\title{
One Perspective on the Value of Journal Editorship
}

\author{
Edith V. Sullivan
}

Received: 28 May 2014 / Accepted: 23 June 2014 / Published online: 16 July 2014

(C) Academic Psychiatry 2014

In 2009, I took the helm of Neuropsychology Review, a quarterly, refereed journal devoted to integrative and synthetic reviews on substantive content areas in neuropsychology. As I saw it, the journal's venue should include scholarly reviews of research on patient populations with endogenous or acquired conditions affecting the brain and its functions and on translational research that can provide a mechanistic understanding of clinical problems. A goal was to steer the reader (and me!) to "break set" and recast our thinking about problems and unknowns with new theories, approaches, and methods that might enhance the understanding of neuropsychological dysfunction. I considered that a major purpose of the journal was to educate scientists, teachers, and students about selective topics in the neurosciences. My aim was to create a resource for all professional levels and specialties of neuropsychology and the neuroscience disciplines from which it emerged and expand it to new areas such as neuroimaging and genetics.

This looked good on paper, but what did I have to do to implement my vision? The steps I took included defining neuropsychology, articulating specific goals for the journal, and recognizing the responsibilities I embraced in accepting this position.

\section{Definition of the Topic}

Neuropsychology is a multifaceted neuroscience discipline, which evolved from neurology, psychiatry, psychology, neurosurgery, and electrophysiology. Historically, its purpose was to localize neural and neurological foundations of selective sensory, motor, and cognitive processes through characterization of selective functions disrupted by circumscribed lesions.

E. V. Sullivan $(\bowtie)$

Stanford University School of Medicine, Stanford, CA, USA

e-mail: edie@stanford.edu
In its current incarnation, neuropsychology reflects the neurosciences and has moved from seeking loci to appreciating multifocal systems and far-reaching neurocircuitry as underlying selective functions. This paradigm shift has required embracing and accommodating highly technical brain imaging modalities and complex analysis approaches. The joint venture of neuropsychology and neuroimaging has launched new levels of questioning and hypothesis testing conducted in vivo, unattainable by neuropsychology's forerunners, whose "imaging" was limited to the neurosurgeon's drawings or to postmortem examination for lesion verification.

As a journal of reviews rather than of primary data, my approach has been to ensure that the topic du jour is covered by the best and most mature researchers of that topic. To enhance chances of achieving this aim, most papers are by invitation; those that are unsolicited suffer an $83 \%$ rejection rate.

To overcome - and perhaps compensate for - a fundamental annoyance I have with journals that limit authors to an arbitrary number of references, my reference allotment is limitless, and I actively encourage authors to expand their reference list where relevant. Similarly, I request that authors use figures in their reviews, which aid in describing neural pathways, neuroimaging findings, selectivity of brain structure-function relations, and functional models. Thorough, thoughtful, synthetic reviews provide an initial place to start learning about a new field. When adequately referenced, supplemented with figures, and written in a scholarly and critical fashion, the relevance, novelty, and unknowns are presented clearly and usefully.

Then I thought about myself: what was my scope and limit of knowledge in "my" field, this expansive multidisciplinary field of neuropsychology? How dare I have the courage-or even the audacity-to assume editorship charging me to judge the worth of scholarly reviews on topics often far afield from my own? 


\section{Goal Setter and Time Keeper}

This source of early-morning awakening on accepting editorship was quelled, but never eradicated, by devising five goals for the journal. To enhance chances of success and to minimize errors in decision-making, I selected a knowledgeable panel of associate editors to assist in making informed judgments and identified editorial board members representing many of the facets of neuropsychology to aid in review.

I published my five goals [1], a ploy for helping me maintained them, and I present an abridged version here (all quotations are from page 2 of the referenced editorial):

1. Put the "neuro" into neuropsychology. Neuroimaging is an essential partner of neuropsychology. Having had the exceptional opportunity to be a neuropsychologist in a neuroimaging laboratory for more than 25 years, I have first-hand learning about imaging modalities, acquisition, image processing, and data interpretation. When coupling quantitative imaging methods with creative neuropsychological tests, double and multiple dissociations can be determined on a continuous scale, serving to identify selective brain regions and systems, either within subjects or between groups, supportive of specific, tested functions. With this background, I could legitimately request, and even demand, that authors put "neuro" into their papers. Fulfilling this goal would ensure that neuropsychologists would go beyond being administrators and interpreters of neuropsychological tests and learn to use and interpret associated brain imaging data. To enrich reviews, I request that authors include relevant images to make their points come alive. In particular, "I propose[d] that any current review of neuropsychological function or dysfunction should include reference to relevant neuroimaging, neurophysiology, neurovascular or other potential neural substrate. Indeed, images from neuroimaging and EEG modalities are encouraged to demonstrate a point. To promote this objective, Springer agreed to print color figures online free of charge."

2. Appreciate new technologies for creative and selective testing. "Successful neuropsychological inquiry is dependent on astute observation of selective functioning and creative testing for selective assessment of functions. ... Not one to chide simplicity in testing (my favorite tool is the mechanical stop watch with a sweep hand), computerized testing has enabled refined questions to be answered regarding speed of thought, movement, and sensory perception; electrophysiological techniques, starting with the astute observations of Fritz and Hitzig (1890) who mapped the motor strip, have provided a vehicle for a legacy of work in epilepsy. The burgeoning fields of biomechanics and virtual reality, for example, afford novel approaches to the behavioral half of the neuropsychology duality."

3. Reprint landmark papers in neuropsychology. "The objective is to appreciate our roots and to move the field forward, lest we cover old ground lacking knowledge of mechanisms and substrates of behavior already identified and some requiring a new context. Internet searches have enabled an unprecedented spread of knowledge through electronic journal and reprint exchange. So often lacking, however, is the seeking of original papers on a topic and an appreciation of initial studies conducted with now considered primitive methods that resulted in defining discoveries."

4. Continue special issues with invited editors for in-depth coverage. "Neuropsychology, with its primary motive of the understanding of brain structure and function, has always been and is more so than ever multidisciplinary. This characteristic makes it nearly impossible for the individual to be expert in all facets of neuropsychological inquiry. Thus, Neuropsychology Review provides a forum through the special issues feature for informing the nonexpert in new technologies and applications." I cannot take credit for conceiving of this successful feature, introduced by my predecessor, Ronald Lazar, Ph.D. Nonetheless, I have continued this approach, which has resulted in high quality, widely read, and cited papers and surely has contributed to the rising impact factor (for 2012 $=6.42$; recent 5 -year impact factor $=7.53$ assessed by Research Gate).

5. Expanded "Aims and Scope" of the journal. "Neuropsychology Review should complement journals that publish new data (Publication of novel data is outside of the purview of Neuropsychology Review.)... by providing synthetic reviews and models of potentially conflicting results. Reviews must be scholarly and provide extensive reference lists....we need thoughtful, responsible reviews to introduce us to new areas and developments, to update us on that which we have not remained current, and to challenge us in our own specialties." The last goal has a special context: Neuropsychology has a curious history, which grew out of multiple, competing disciplines and competing scientists. Given this genesis, there is little surprise that this field is riddled with factions. Thoughtful argument and differences are healthy, energizing, and essential for innovation, whereas factious bastions do little toward this end. Accordingly, I have actively invited thoughtful argument and welcomed stridently, clearly, and fairly argued differences for consideration for publication. The intended outcome is to educate the readership on the tenets and bases of differences thereby affording the chance for understanding differences, identifying common ground, and determining whether and how opposing camps of thought can logically co-exist. 


\section{What Value Has Editorship of a Journal Contributed to My Professional Life?}

Editorship offered me a rich source of challenge, opportunity, and professional growth. My hope has been to create a forum for education and opportunity for advanced scientists to explicate positions and present synthetic reviews and models that evolved from rigorous research. I quickly learned that editorship is a privilege with obligations to the publisher for timely and punctual finished products; commitment to the readership for thoughtful, accurate, and informative reviews; a responsibility to authors for quick, thorough, and fair review; and opportunity for expansion of the scientist, student, and field. The journal has enriched my professional purview, broadened my scientific knowledge to include topics I had never recognized, and brought me satisfaction in its success. I am also proud of the writing (I spend countless hours editing manuscripts for grammar, diction, and content), presentation, and its look. I fought and won a battle with my managing editor to produce a new cover with each new issue. (I invite you to go to the website to explore the special issues and enjoy their special cover images: http://link. springer.com/journal/volumesAndIssues/11065.)

I am not done yet! Remaining to be completed is the development of four more themes for the last four issues of my tenure as editor-in-chief. What have we not yet covered that neuropsychologists and other neuroscientists should know about to displace or upend fossilized thought or stance about brain structure and its many complex functions? I hope that the contributors and consumers of Neuropsychology Review under my watch have gained as much as I have in developing each issue.

Implications for Academic Leaders

- Define your audience and provide the highest quality information in the most interesting way to your audience, whom you must assume comes as a willing and educable student.

- Have the courage to make informed decisions, whether popular or unpopular, whether positive or negative, whether of the majority or the minority vote, and, as importantly, have the courage to change your position when an adequate argument is presented.

Acknowledgments The writing of this essay was supported by grants to the author from the National Institute on Alcohol Abuse and Alcoholism (AA010723, AA017168).

Disclosures I am the editor-in-chief for Neuropsychology Review, and I receive a stipend for this work.

\section{Reference}

1. Sullivan EV. Neuropsychology review: a resource for the clinical, experimental, and translational neuropsychologists. Neuropsychol Rev. 2009;19:1-3. 